\title{
A Stochastic Lotka-Volterra Competitive System With Feedback Controls
}

\author{
X.J. Dai \\ Science College of Hunan Agriculture University \\ Changsha, 410128, PR China
}

\begin{abstract}
In this paper, we consider an autonomous LotkaVolterra competitive system with stochastic perturbation and feedback controls. Firstly, we show the existence, the uniqueness and the positivity of the solution. Secondly, under a simple assumption, sufficient conditions for stability in the mean and extinction of each population are established.
\end{abstract}

Keywords-feedback controls; competitive system; stochastic perturbation; extinction; stability in the mean

\section{INTRODUCTION}

For the last decades, the classical Lotka-Volterra competition system has been studied extensively. Many excellent results are obtained (see $[13,14])$.

In [1], the authors argued that in a situation where the equilibrium is not the desirable one (or affordable) and a smaller value is required, we are required to alter the system structurally by introducing a feedback control variable [2] . This can be implemented by means of a biological control or some harvesting procedure so as to make the population stabilize at a lower value. In 1931 V. Volterra explained the balance between two populations of fish in a closed pond using the theory of feedback. Later, a series of mathematical models have been established to describe the dynamics of feedback control systems.

Gopalsamy and Weng [3] introduced a two species autonomous Lotka-Volterra competitive system with feedback controls.

Where ${ }^{x_{i}(t)}$ denotes the population density, ${ }_{i}(t)$ denotes the feedback control variable, and $r_{i}, a_{i j} c_{i}, e_{i}, d_{i}(i, j=1,2)$ are positive constants.

$$
\left\{\begin{array}{l}
d x_{1}(t)=x_{1}(t)\left[r_{1}-a_{11} x_{1}(t)-a_{12} x_{2}(t)-c_{1} u_{1}(t)\right] d t \\
d x_{2}(t)=x_{2}(t)\left[r_{2}-a_{21} x_{1}(t)-a_{22} x_{2}(t)-c_{2} u_{2}(t)\right] d t \\
d u_{1}(t)=\left[-e_{1} u_{1}(t)+d_{1} x_{1}(t)\right] d t \\
d u_{2}(t)=\left[-e_{2} u_{2}(t)+d_{2} x_{2}(t)\right] d t .
\end{array}\right.
$$

They obtained sufficient conditions for the globally asymptotically stable of system (1).

But, in the real world population systems often subject to environmental perturbations. In many cases, these perturbations should not be neglected, and there are many authors have introduced stochastic population models in order to investigate the effect of environmental noises; see (e.g. [4, 6-10, 12]). For example, Meng Liu, Ke Wang [9] discussed a stochastic competition system

\author{
X.P. Li*
}

Science College of Hunan Agriculture University Changsha, 410128, PR China

$\left\{\begin{array}{l}\mathrm{d} x_{1}(t)=x_{1}(t)\left[r_{1}-a_{11} x_{1}(t)-a_{12} x_{2}(t)\right] d t+\sigma_{1} x_{1}(t) d B_{1}(t) \\ \mathrm{d} x_{2}(t)=x_{2}(t)\left[r_{2}-a_{21} x_{1}(t)-a_{22} x_{2}(t)\right] d t+\sigma_{2} x_{2}(t) d B_{2}(t) .\end{array}\right.$

Where $r_{i}, a_{i j}, \sigma_{i}(i, j=1,2$ ) are positive constants.

However, to this day, no scholar has investigated the dynamic behaviors of the stochastic Lotka-Volterra competitive system with feedback controls. In this paper, we consider a stochastic Lotka-Volterra competitive system with feedback controls. Suppose that the environmental noises mainly affect the growth rate $r_{i}, r_{i}=r_{i}+\sigma_{i} d B_{i}(t)$, (see [6-10]), where $\sigma_{i}^{2}$ denotes the intensity of the noise, and $B_{i}(t)$ is a standard Brownian motion defined on a complete probability space $(\Omega, F, P), i=1,2$. Then we get following stochastic population system

$$
\left\{\begin{array}{l}
\mathrm{d} x_{1}(t)=x_{1}(t)\left[r_{1}-a_{11} x_{1}(t)-a_{12} x_{2}(t)-c_{1} u_{1}(t)\right] d t+\sigma_{1} x_{1}(t) d B_{1}(t) \\
\mathrm{d} x_{2}(t)=x_{2}(t)\left[r_{2}-a_{21} x(t)-a_{22} y(t)-c_{2} u_{2}(t)\right] d t+\sigma_{2} x_{2}(t) d B_{2}(t) \\
d u_{1}(t)=\left[-e_{1} u_{1}(t)+d_{1} x_{1}(t)\right] d t \\
d u_{2}(t)=\left[-e_{2} u_{2}(t)+d_{2} x_{2}(t)\right] d t
\end{array}\right.
$$

With initial conditions $x_{i}(0)>0, u_{i}(0)>0, \quad i=1,2$.

We can easy to see that system (3) has no positive equilibrium. Hence, there is an interesting and important question is that of whether system (3) still has some stability around some positive point. In this paper, we shall show it.

\section{MAIN RESULTS}

In this section, we first show that the solution of system (3) is global and positive.

Theorem 2.1 For any given initial value $\left(x_{1}(0), x_{2}(0), u_{1}(0), u_{2}(0)\right) \in R_{+}^{4}$, system (3) has a unique positive solution $\left(x_{1}(t), x_{2}(t), u_{1}(t), u_{2}(t)\right)$ on $t \geq 0$ and the solution will remain in $R_{+}^{4}$ with probability 1 , where

$$
\begin{array}{cr}
R_{+}^{4}=\left\{\left(x_{1}, x_{2}, u_{1}, u_{2}\right) \in R^{4} \mid x_{i}>0, u_{i}>0, i=1,2\right\} & \\
\text { Proof } \quad \cdot \quad \text { Define a dunction } \\
V\left(x_{1}, x_{2}, u_{1}, u_{2}\right)=\sum_{i=1}^{2}\left(x_{i}-1-\ln x_{i}\right)+\sum_{i=1}^{2} e_{i}^{-1} d_{i}\left(u_{i}-1-\ln u_{i}\right) \quad, \quad \text { if } \\
\left(x_{1}(t), x_{2}(t), u_{1}(t), u_{2}(t)\right) \in R_{+}^{4} \text {, we obtain that }
\end{array}
$$




$$
\begin{aligned}
d V\left(x_{1}, x_{2}, u_{1}, u_{2}\right)= & \left(x_{1}-1\right)\left[r_{1}-a_{11} x_{1}-a_{12} x_{2}-c_{1} u_{1}\right] d t+0.5 \sigma_{1}^{2} d t+\left(x_{1}-1\right) \sigma_{1} d B_{1}(t) \\
& +\left(x_{2}-1\right)\left[r_{2}-a_{21} x_{1}-a_{22} x_{2}-c_{2} u_{2}\right] d t+0.5 \sigma_{2}^{2} d t+\left(x_{2}-1\right) \sigma_{2} d B_{2}(t) \\
& -c_{1} u_{1}+c_{1}+e_{1}^{-1} c_{1} d_{1} x_{1}-d_{1} x_{1} u_{1}^{-1}-c_{2} u_{2}+c_{2}+e_{2}^{-1} c_{2} d_{2} x_{2}-d_{2} x_{2} u_{2}^{-1} \\
\leq & {\left[\left(r_{1}+a_{11}+a_{21}+e_{1}^{-1} c_{1} d_{1}\right) x_{1}-a_{11} x_{1}^{2}+\left(r_{2}+a_{22}+a_{12}+e_{2}^{-1} c_{2} d_{2}\right) x_{2}-a_{22} x_{2}^{2}\right.} \\
& \left.+0.5 \sigma_{1}^{2}+0.5 \sigma_{2}^{2}+c_{1}+c_{2}\right] d t+\left(x_{1}-1\right) \sigma_{1} d B_{1}(t)+\left(x_{2}-1\right) \sigma_{2} d B_{2}(t) \\
\leq & K+\left(x_{1}-1\right) \sigma_{1} d B_{1}(t)+\left(x_{2}-1\right) \sigma_{2} d B_{2}(t) .
\end{aligned}
$$

Where $K$ is a positive constant. By the similar proof of [12, Theorem 2.1], we can obtain the desired assertion.

For simplicity, we introduce the following notation. Let

$$
\begin{gathered}
\Delta_{1}=\left(a_{22}+\frac{c_{2} d_{2}}{e_{2}}\right)\left(r_{1}-0.5 \sigma_{1}^{2}\right)-\left(r_{2}-0.5 \sigma_{2}^{2}\right) a_{12} \\
\Delta_{2}=\left(a_{11}+\frac{c_{1} d_{1}}{e_{1}}\right)\left(r_{2}-0.5 \sigma_{2}^{2}\right)-\left(r_{1}-0.5 \sigma_{1}^{2}\right) a_{21} \\
\Delta=\left(a_{11}+\frac{c_{1} d_{1}}{e_{1}}\right)\left(a_{22}+\frac{c_{2} d_{2}}{e_{2}}\right)-a_{12} a_{21} \\
f^{*}=\lim _{t \rightarrow+\infty} \sup f(t) \\
f_{*}=\lim _{t \rightarrow+\infty} \inf f(t) \\
\langle f(t)\rangle=t^{-1} \int_{0}^{t} f(s) d s
\end{gathered}
$$

Theorem 2.2 (I) If $r_{1}<0.5 \sigma_{1}^{2}$ and $r_{2}<0.5 \sigma_{2}^{2}$, then both $x_{1}$ and $^{x_{2}}$ go to extinction almost surely (a.s.),ie.

(II) If $r_{i}>0.5 \sigma_{i}^{2}$ and $r_{j}<0.5 \sigma_{j}^{2}$, then $x_{j}$ goes to extinction a.s. and $x_{i}$ is stable in time average a.s. $i, j=1,2, \quad i \neq j$,

$$
\begin{gathered}
\lim _{t \rightarrow+\infty} t^{-1} \int_{0}^{t} x_{i}(s) d s=\frac{\left(r_{i}-0.5 \sigma_{i}^{2}\right) e_{i}}{a_{i i} e_{i}+c_{i} d_{i}} \\
\lim _{t \rightarrow+\infty} t^{-1} \int_{0}^{t} u_{i}(s) d s=\frac{\left(r_{i}-0.5 \sigma_{i}^{2}\right) d_{i}}{a_{i i} e_{i}+c_{i} d_{i}} \text { a.s. }
\end{gathered}
$$

(III) If $r_{1}>0.5 \sigma_{1}^{2}$ and $r_{2}>0.5 \sigma_{2}^{2}$.

(1) Suppose that $\Delta>0$ (It is easy to see that $\Delta_{1}<0$ and $\Delta_{2}<0$ cannot simultaneously hold in this case).

(a) If $\Delta_{i}>0$ and $\Delta_{j}<0$, then $x_{j}$ goes to extinction a.s. and $x_{i}$ is stable in time average a.s., $i, j=1,2, i \neq j$, $\lim _{t \rightarrow+\infty} t^{-1} \int_{0}^{t} x_{i}(s) d s=\frac{\left(r_{i}-0.5 \sigma_{i}^{2}\right) e_{i}}{a_{i i} e_{i}+c_{i} d_{i}}, \lim _{t \rightarrow+\infty} t^{-1} \int_{0}^{t} u_{i}(s) d s=\frac{\left(r_{i}-0.5 \sigma_{i}^{2}\right) d_{i}}{a_{i i} e_{i}+c_{i} d_{i}}$ a.s.

(b) If $\Delta_{1}>0$ and $\Delta_{2}>0$, then both $x_{1}$ and $x_{2}$ are stable in time average a.s.,

$\lim _{t \rightarrow+\infty} t^{-1} \int_{0}^{t} x_{i}(s) d s=\frac{\Delta_{i}}{\Delta}, \lim _{t \rightarrow+\infty} t^{-1} \int_{0}^{t} u_{i}(s) d s=\frac{\Delta_{i} d_{i}}{\Delta e_{i}}$ a.s. $i=1,2$.

(2) Suppose that $\Delta<0$ (It is easy to see that $\Delta_{1}>0$ and $\Delta_{2}>0$ cannot simultaneously hold in this case).

(a) If $\Delta_{i}<0$ and $\Delta_{j}>0$, then $x_{i}$ goes to extinction a.s. and $^{x_{j}}$ is stable in time average a.s., $i, j=1,2, i \neq j$, $\lim _{t \rightarrow \infty} t^{-1} \int_{0}^{t} x_{j}(s) d s=\frac{\left(r_{j}-0.5 \sigma_{j}^{2}\right) e_{j}}{a_{i j} e_{j}+c_{j} d_{j}}, \lim _{t \rightarrow+\infty} t^{-1} \int_{0}^{t} u_{j}(s) d s=\frac{\left(r_{j}-0.5 \sigma_{j}^{2}\right) d_{j}}{a_{i j} e_{j}+c_{j} d_{j}}$ a.s.

(b) If $\Delta_{1}<0$ and $\Delta_{2}<0$, then $x_{1}$ and $x_{2}$ not simultaneously go to extinction (a.s).

\section{PROOF OF THE THEOREM 2.2}

Firstly, we introduce some fundamental lemmas which will be used.

Lemma 3.1 ([7]). Suppose that $z(t) \in C\left(\Omega \times R_{+}\right)$,

(i) If there exist two positive constants $\mathrm{T}$, and $\mathrm{u}$ such that

$$
\ln z(t) \leq \lambda t-u \int_{0}^{t} z(s) d s+\sum_{i=1}^{n} \alpha_{i} B_{i}(t)
$$

For all $t \geq T$, where $\alpha_{i}(i=1,2)$ are constants, then

$$
\left\{\begin{array}{l}
\langle z(t)\rangle^{*} \leq u^{-1} \lambda, \text { a.s. if } \lambda \geq 0 \\
\lim _{t \rightarrow \infty} z(t)=0, \text { a.s. if } \lambda<0 .
\end{array}\right.
$$

(ii) If there exist three positive constants, $\lambda, \mathrm{T}$ and $\mathrm{u}$ such that $\ln x(t) \geq \lambda t-u \int_{0}^{t} x(s) d s+\sum_{i=1}^{n} \alpha_{i} B_{i}(t)$, for all $t \geq T$, then $\langle z(t)\rangle_{*} \geq u^{-1} \lambda$, a.s.

Lemma 3.2 (see [11]). Consider one-dimensional stochastic differential equation

$$
d x=x[a-b x] d t+\sigma x d B(t)
$$

where $\mathrm{a}, \mathrm{b}, \sigma$ are positive, and $\mathrm{B}(\mathrm{t})$ is standard Brownian motion, Under the condition $a>0.5 \sigma^{2}$, for any initial value $x_{0}>0$, the solution $\mathrm{x}(\mathrm{t})$ to (5) has the properties $\lim _{t \rightarrow+\infty} t^{-1} \ln x(t)=0$, a.s.

Lemma 3.3 If $r_{i} \neq 0.5 \sigma_{i}^{2}$, for any initial value $\left(x_{1}(0), x_{2}(0), u_{1}(0), u_{2}(0)\right) \in R_{+}^{4}$, for $x_{i}(t), u_{i}(t)$, of system (3) have $\left[t^{-1} \ln x_{i}(t)\right] \leq 0, \lim _{t \rightarrow \infty} t^{-1} u_{i}(t)=0$, a.s. $i=1,2$.

Proof. From (3), it is obvious that $d x_{i}(t) \leq x_{i}(t)\left[r_{i}-a_{i i} x_{i}(t)\right] d t+\sigma_{i} x_{i}(t) d B_{i}(t)$.

Denote $X_{i}(t)$ is the solution to the following stochastic equation

$$
\left\{\begin{array}{l}
\mathrm{d} X_{i}(t)=X_{i}(t)\left[r_{i}-a_{i i} X_{i}(t)\right] d t+\sigma_{i} X_{i}(t) d B_{i}(t) \\
X_{i}(0)=x_{i}(0) \quad i=1,2
\end{array}\right.
$$

Then $X_{i}(t)$ have the following explicit representations respectively

$$
X_{i}(t)=\frac{\exp \left\{r_{i} t-0.5 \sigma_{i}^{2} t+\sigma_{i} B_{i}(t)\right\}}{x_{i}^{-1}(0)+a_{i i} \int_{0}^{t} \exp \left\{r_{i} s-0.5 \sigma_{i}^{2} s+\sigma_{i} B_{i}(s)\right\} d s}, \quad i=1,2 .(6)
$$


From the third and fourth equations of system (3), we can obtain $u_{i}(t)$ have the following explicit representations respectively $u_{i}(t)=\exp \left\{-e_{i} t\right\}\left[d_{i} \int_{0}^{t} x(s) \exp \left\{e_{i} s\right\} d s+u_{i}(0)\right]$.

If $r_{i}<0.5 \sigma_{i}^{2}$, then from (6) we have

$$
x_{i}(t) \leq X_{i}(t) \leq X_{i}(0) \exp \left\{-\left[0.5 \sigma_{i}^{2}-r_{i}-\sigma_{i} t^{-1} B_{i}(t)\right] t\right\}
$$

Because of $\lim _{t \rightarrow+\infty} t^{-1} B_{i}(t)=0$ a.s. $\lim _{t \rightarrow+\infty} x_{i}(t)=0$ and $\left[t^{-1} \ln x_{i}(t)\right]^{*} \leq 0$ a.s. , obviously, $\lim _{t \rightarrow+\infty} t^{-1} u_{i}(t)=0$ a.s. $i=1,2$. If ${ }^{r_{i}}>0.5 \sigma_{i}^{2}$, by virtue of Lemma 3.2 we have

$$
\lim _{t \rightarrow+\infty} t^{-1} \ln x_{i}(t) \leq \lim _{t \rightarrow+\infty} t^{-1} \ln X_{i}(t)=0 \text { a.s. } i=1,2 .
$$

For $\exp \left(e_{i} t\right) \ln X_{i}(t)$ apply Itô's formula, we can see that $a_{i j} \int_{0}^{t} \exp \left(e_{i} s\right) X_{i}(s) d s=\ln X_{i}(0)-\exp \left(e_{i} t\right) \ln X_{i}(t)+e_{i} \int_{0}^{t} \exp \left(e_{i} s\right) \ln X_{i}(s) d s$ $+\int_{0}^{t} \exp \left(e_{i} s\right)\left(r_{i}-0.5 \sigma_{i}^{2}\right) d s+\sigma_{i} \int_{0}^{t} \exp \left\{e_{i} s\right\} d B_{i}(s)$.

Using mean value theorem of integrals, we obtain

$$
\begin{aligned}
\frac{a_{i i} \int_{0}^{t} \exp \left(e_{i} s\right) X_{i}(s) d s}{t \exp \left(e_{i} t\right)}=\frac{\ln X_{i}(0)}{t \exp \left(e_{i} t\right)}-\frac{\ln X_{i}(t)}{t}+\frac{e_{i} \ln X_{i}\left(\tau_{1}\right) \int_{0}^{t} \exp \left(e_{i} s\right) d s}{t \exp \left(e_{i} t\right)} \\
+\frac{\int_{0}^{t} \exp \left(e_{i} s\right)\left(r_{i}-0.5 \sigma_{i}^{2}\right) d s}{t \exp \left(e_{i} t\right)}+\frac{\sigma_{i}\left[\exp \left\{e_{i} t\right\} B_{i}(t)-e_{i} B_{i}\left(\tau_{2}\right) \int_{0}^{t} \exp \left\{e_{i} s\right\} d s\right]}{t \exp \left(e_{i} t\right)} \\
\quad \leq t^{-1} \ln X_{i}(0)-t^{-1} \ln X_{i}(t)+t^{-1} \ln X_{i}\left(\tau_{1}\right)-t^{-1} \exp \left\{-e_{i} t\right\} \ln X_{i}\left(\tau_{1}\right) \\
\quad+\left(e_{i} t\right)^{-1}\left(r_{i}-0.5 \sigma_{i}^{2}\right)+t^{-1} \sigma_{i} B_{i}(t)-t^{-1} \sigma_{i} B_{i}\left(\tau_{2}\right)+t^{-1} \exp \left\{-e_{i} t\right\} \sigma_{i} B_{i}\left(\tau_{2}\right)
\end{aligned}
$$

Where $\tau_{i} \in[0, t], i=1,2$. By virtue of Lemma 3.2 and $\lim t^{-1} B_{i}(t)=0$

$\lim _{t \rightarrow+\infty} t^{-1} B_{i}(t)=0$, then we can get

$0 \leq \lim _{t \rightarrow+\infty} \frac{d_{i} \int_{0}^{t} \exp \left(e_{i} s\right) x_{i}(s) d s+u_{i}(0)}{t \exp \left(e_{i} t\right)} \leq \lim _{t \rightarrow+\infty} \frac{d_{i} \int_{0}^{t} \exp \left(e_{i} s\right) X_{i}(s) d s+u_{i}(0)}{t \exp \left(e_{i} t\right)}=0$ a.s.

Therefore, $\lim _{t \rightarrow+\infty} t^{-1} u_{i}(t)=0$ a.s. $i=1,2$.

Proof of Theorem 2.2. Applying Itô's formula to (3), we can see that

$$
\begin{gathered}
t^{-1} \ln x_{1}(t) / x_{1}(0)=\left(r_{1}-0.5 \sigma_{1}^{2}\right)-a_{11}\left\langle x_{1}(t)\right\rangle-a_{12}\left\langle x_{2}(t)\right\rangle-c_{1}\left\langle u_{1}(t)\right\rangle+t^{-1} \sigma_{1} B_{1}(t)(7) \\
t^{-1} \ln x_{2}(t) / x_{2}(0)=\left(r_{2}-0.5 \sigma_{2}^{2}\right)-a_{21}\left\langle x_{1}(t)\right\rangle-a_{22}\left\langle x_{2}(t)\right\rangle-c_{2}\left\langle u_{2}(t)\right\rangle+t^{-1} \sigma_{2} B_{2}(t)(8) \\
t^{-1}\left[u_{1}(t)-u_{1}(0)\right]=-e_{1}\left\langle u_{1}(t)\right\rangle+d_{1}\left\langle x_{1}(t)\right\rangle \\
t^{-1}\left[u_{2}(t)-u_{2}(0)\right]=-e_{2}\left\langle u_{2}(t)\right\rangle+d_{2}\left\langle x_{2}(t)\right\rangle
\end{gathered}
$$$$
\text { (I): Suppose that } r_{1}<0.5 \sigma_{1}^{2} \text { and } r_{2}<0.5 \sigma_{2}^{2} \text {, by virtue of }
$$
Lemma 3.3's proof we can obtain $\lim _{t \rightarrow+\infty} x_{i}(t)=0, \lim _{t \rightarrow+\infty} u_{i}(t)=0$ a.s. $i=1,2$

(II): Without loss of generality, Suppose that $r_{1}>0.5 \sigma_{1}^{2}$ and $r_{2}<0.5 \sigma_{2}^{2}$. Since ${ }^{r_{2}<0.5 \sigma_{2}^{2}}$, then by (I) we can get, $\lim _{t \rightarrow+\infty} x_{2}(t)=0$ and $\lim _{t \rightarrow+\infty} u_{2}(t)=0$. for arbitrary $\varepsilon>0$,
There exist a positive constant $\mathrm{T}$ such that for $\mathrm{t}>\mathrm{T}$,

$$
\begin{gathered}
-\varepsilon / 3 \leq a_{12}\left\langle x_{2}(t)\right\rangle \leq \varepsilon / 3 \\
-\varepsilon / 3 \leq t^{-1} \ln x_{2}(0) \leq \varepsilon / 3 \\
-\varepsilon / 3 \leq c_{1}\left(e_{1} t\right)^{-1}\left[u_{1}(t)-u_{1}(0)\right] \leq \varepsilon / 3
\end{gathered}
$$

Thus computing $(7)-e_{1}^{-1} c_{1} d_{1} \times(9)$, we have

$$
t^{-1} \ln x_{1}(t) \leq\left(r_{1}-0.5 \sigma_{1}^{2}+\varepsilon\right)-\left(a_{11}+e_{1}^{-1} c_{1} d_{1}\right)\left\langle x_{1}(t)\right\rangle+t^{-1} \sigma_{1} B_{1}(t)
$$

$t^{-1} \ln x_{1}(t) \geq\left(r_{1}-0.5 \sigma_{1}^{2}-\varepsilon\right)-\left(a_{11}+e_{1}^{-1} c_{1} d_{1}\right)\left\langle x_{1}(t)\right\rangle+t^{-1} \sigma_{1} B_{1}(t)$

Since $r_{1}>0.5 \sigma_{1}^{2}$, we can choose $\varepsilon$ sufficiently small such that $r_{1}-0.5 \sigma_{1}^{2}-\varepsilon>0$,

Apply (i) and (ii) of Lemma 3.1 to above inequalities, we obtain

$$
\frac{\left(r_{1}-0.5 \sigma_{1}^{2}-\varepsilon\right) e_{1}}{a_{11} e_{1}+c_{1} d_{1}} \leq\left\langle x_{1}(t)\right\rangle_{*} \leq\left\langle x_{1}(t)\right\rangle^{*} \leq \frac{\left(r_{1}-0.5 \sigma_{1}^{2}+\varepsilon\right) e_{1}}{a_{11} e_{1}+c_{1} d_{1}} \quad \text { a.s. }
$$

An application of the arbitrariness of $\varepsilon$ leads to

$$
\begin{aligned}
& \lim _{t \rightarrow+\infty} t^{-1} \int_{0}^{t} x_{1}(s) d s=\frac{\left(r_{1}-0.5 \sigma_{1}^{2}\right) e_{1}}{a_{11} e_{1}+c_{1} d_{1}}, \lim _{t \rightarrow+\infty} t^{-1} \int_{0}^{t} u_{1}(s) d s=\frac{\left(r_{1}-0.5 \sigma_{1}^{2}\right) d_{1}}{a_{11} e_{1}+c_{1} d_{1}} \text { a.s. } \\
& \text { Now let us prove (III), computing }(7)-\frac{c_{1}}{e_{1}} \times(9) \text { and } \\
& \text { (8) }-\frac{c_{2}}{e_{2}} \times(10) \text { we have }
\end{aligned}
$$

$t^{-1} \ln x_{1}(t) / x_{1}(0)-\left(e_{1} t\right)^{-1} c_{1}\left[u_{1}(t)-u_{1}(0)\right]=\left(r_{1}-\frac{\sigma_{1}^{2}}{2}\right)-\left(a_{11}+\frac{c_{1} d_{1}}{e_{1}}\right)\left\langle x_{1}\right\rangle-a_{12}\left\langle x_{2}\right\rangle+t^{-1} \sigma_{1} B_{1}(t)$

$t^{-1} \ln x_{2}(t) / x_{2}(0)-\left(e_{2} t\right)^{-1} c_{2}\left[u_{2}(t)-u_{2}(0)\right]=\left(r_{2}-\frac{\sigma_{2}^{2}}{2}\right)-\left(a_{22}+\frac{c_{2} d_{2}}{e_{2}}\right)\left\langle x_{2}\right\rangle-a_{21}\left\langle x_{1}\right\rangle+t^{-1} \sigma_{2} B_{2}(t)$

for arbitrary $\varepsilon>0$, There exist a positive constant $\mathrm{T}$, such that for $\mathrm{t}>\mathrm{T}$,

$$
-\varepsilon / 2 \leq t^{-1} \ln x_{i}(0) \leq \varepsilon / 2, \quad-\varepsilon / 2 \leq c_{i}\left(e_{i} t\right)^{-1}\left[u_{i}(t)-u_{i}(0)\right] \leq \varepsilon / 2, i=1,2(15)
$$

Substituting the above inequalities into (13) and (14), one can obtain that for $t>T$, we have

$t^{-1} \ln x_{1}(t) \leq\left(r_{1}-0.5 \sigma_{1}^{2}+\varepsilon\right)-\left(a_{11}+e_{1}^{-1} c_{1} d_{1}\right)\left\langle x_{1}(t)\right\rangle-a_{12}\left\langle x_{2}(t)\right\rangle+t^{-1} \sigma_{1} B_{1}(t)(16)$
$t^{-1} \ln x_{1}(t) \geq\left(r_{1}-0.5 \sigma_{1}^{2}-\varepsilon\right)-\left(a_{11}+e_{1}^{-1} c_{1} d_{1}\right)\left\langle x_{1}(t)\right\rangle-a_{12}\left\langle x_{2}(t)\right\rangle+t^{-1} \sigma_{1} B_{1}(t)(17)$
$t^{-1} \ln x_{2}(t) \leq\left(r_{2}-0.5 \sigma_{2}^{2}+\varepsilon\right)-\left(a_{22}+e_{2}^{-1} c_{2} d_{2}\right)\left\langle x_{2}(t)\right\rangle-a_{21}\left\langle x_{1}(t)\right\rangle+t^{-1} \sigma_{2} B_{2}(t)(18)$
$t^{-1} \ln x_{2}(t) \geq\left(r_{2}-0.5 \sigma_{2}^{2}-\varepsilon\right)-\left(a_{22}+e_{2}^{-1} c_{2} d_{2}\right)\left\langle x_{2}(t)\right\rangle-a_{21}\left\langle x_{1}(t)\right\rangle+t^{-1} \sigma_{2} B_{2}(t) .(19)$

Computing $(16) \times\left(a_{22}+e_{2}^{-1} c_{2} d_{2}\right)-(19) \times a_{12}$, by virtue of Lemma 3.3, we can obtain

$$
\begin{aligned}
& \left(a_{22}+e_{2}^{-1} c_{2} d_{2}\right) t^{-1} \ln x_{1}(t) \leq\left(a_{22}+e_{2}^{-1} c_{2} d_{2}\right)\left(r_{1}-0.5 \sigma_{1}^{2}+\varepsilon\right)-a_{12}\left(r_{2}-0.5 \sigma_{2}^{2}-\varepsilon\right) \\
& -\Delta\left\langle x_{1}(t)\right\rangle+\left(e_{2} t\right)^{-1}\left(a_{22} e_{2}+c_{2} d_{2}\right) \sigma_{1} B_{1}(t)-t^{-1} a_{21} \sigma_{2} B_{2}(t), \\
& \text { For } \mathrm{t}>\mathrm{T}, \text { at the same time, } \\
& \text { computing }(18) \times\left(a_{11}+e^{-1} c_{1} d_{1}\right)-(17) \times a_{21} \text {, by virtue of } \\
& \text { Lemma 3.3, we can obtain }
\end{aligned}
$$


$\left(a_{11}+e^{-1} c_{1} d_{1}\right) t^{-1} \ln x_{2}(t) \leq\left(a_{11}+e^{-1} c_{1} d_{1}\right)\left(r_{2}-0.5 \sigma_{2}^{2}+\varepsilon\right)-a_{21}\left(r_{1}-0.5 \sigma_{1}^{2}-\varepsilon\right)$

$$
-\Delta\left\langle x_{2}(t)\right\rangle+\left(e_{1} t\right)^{-1}\left(a_{11} e_{1}+c_{1} d_{1}\right) \sigma_{2} B_{2}(t)-t^{-1} a_{21} \sigma_{1} B_{1}(t) .
$$

Case (1) :( a) Without loss of generality. Suppose $\Delta_{1}>0$ and $\Delta_{2}<0$, then let $\varepsilon$ be sufficiently small such that $\left(a_{11}+e_{1}^{-1} c_{1} d_{1}\right)\left(r_{2}-0.5 \sigma_{2}^{2}+\varepsilon\right)-\left(r_{1}-0.5 \sigma_{1}^{2}-\varepsilon\right) a_{21}<0$, applying

(i) of lemma 3.1 to (21) have $\lim _{t \rightarrow+\infty} x_{2}(t)=0, \lim _{t \rightarrow+\infty} u_{2}(t)=0$ a.s. then the proof of

$\lim _{t \rightarrow+\infty} t^{-1} \int_{0}^{t} x_{1}(s) d s=\frac{\left(r_{1}-0.5 \sigma_{1}^{2}\right) e_{1}}{a_{11} e_{1}+c_{1} d_{1}}, \quad \lim _{t \rightarrow+\infty} t^{-1} \int_{0}^{t} u_{1}(s) d s=\frac{\left(r_{1}-0.5 \sigma_{1}^{2}\right) d_{1}}{a_{11} e_{1}+c_{1} d_{1}} \quad$ a.s. is similar to that of (II) and hence is omitted.

(b) Suppose $\Delta_{1}>0$ and $\Delta_{2}>0$. Since $\Delta_{1}>0$, by virtue of Lemma 3.1 (i), (20) and the arbitrariness of $\varepsilon$, we can obtain

$$
\left\langle x_{1}(t)\right\rangle^{*} \leq \frac{\left(a_{22}+e_{2}^{-1} c_{2} d_{2}\right)\left(r_{1}-0.5 \sigma_{1}^{2}\right)-a_{12}\left(r_{2}-0.5 \sigma_{2}^{2}\right)}{\Delta}=\frac{\Delta_{1}}{\Delta},
$$

Similarly, by virtue of (21), Lemma 3.1 (i) and the arbitrariness of ${ }^{\mathcal{E}}$, we have

$$
\left\langle x_{2}(t)\right\rangle^{*} \leq \frac{\Delta_{2}}{\Delta} \quad \text { a.s. }
$$

Let $\quad \varepsilon \quad$ sufficiently small such that $\left(a_{11}+\frac{c_{1} d_{1}}{e_{1}}\right) \frac{\Delta_{1}}{\Delta}-\varepsilon>0$.

we have

$$
\begin{gathered}
t^{-1} \ln x_{1}(t) \geq\left(r_{1}-0.5 \sigma_{1}^{2}-\varepsilon\right)-a_{12} \frac{\Delta_{2}}{\Delta}-\left(a_{11}+\frac{c_{1} d_{1}}{e_{1}}\right)\left\langle x_{1}(t)\right\rangle+t^{-1} \sigma_{1} B_{1}(t) \\
\geq\left(a_{11}+\frac{c_{1} d_{1}}{e_{1}}\right) \frac{\Delta_{1}}{\Delta}-\varepsilon-\left(a_{11}+\frac{c_{1} d_{1}}{e_{1}}\right)\left\langle x_{1}(t)\right\rangle+t^{-1} \sigma_{1} B_{1}(t) .
\end{gathered}
$$

Applying (ii) of lemma 3.1 to above inequality and making use of the arbitrariness of ${ }^{\varepsilon}$, we have

$$
\left\langle x_{1}(t)\right\rangle_{*} \geq \frac{\Delta_{1}}{\Delta} \quad \text { a.s. }
$$

Similarly, leads to $\left\langle x_{2}(t)\right\rangle_{*} \geq \frac{\Delta_{2}}{\Delta}$ a.s. together with (22)(24), have

$$
\lim _{t \rightarrow+\infty}\left\langle x_{i}\right\rangle=\frac{\Delta_{i}}{\Delta}, \quad \text { a.s. } \lim _{t \rightarrow+\infty}\left\langle u_{i}\right\rangle=\frac{\Delta_{i} d_{i}}{\Delta e_{i}}, \quad \text { a.s. } i=1,2 ;
$$

Case (2): (a) without loss of generality. $\operatorname{Suppose}^{\Delta_{1}<0}$ and $^{\Delta_{2}>0}$.

Computing $(19) \times\left(a_{11}+\frac{c_{1} d_{1}}{e_{1}}\right)-(16) \times a_{21}$, have

$\left(a_{11}+\frac{c_{1} d_{1}}{e_{1}}\right) t^{-1} \ln x_{2}(t) \geq t^{-1} a_{21} \ln x_{1}(t)+\left(a_{11}+\frac{c_{1} d_{1}}{e_{1}}\right)\left(r_{2}-0.5 \sigma_{2}^{2}-\varepsilon\right)-a_{21}\left(r_{1}-0.5 \sigma_{1}^{2}+\varepsilon\right)$

$$
-\Delta\left\langle x_{2}(t)\right\rangle+\left(a_{11} e_{1}+c_{1} d_{1}\right) \sigma_{2}\left(t e_{1}\right)^{-1} B_{2}(t)-a_{21} \sigma_{1} t^{-1} B_{1}(t) .
$$

$$
\begin{array}{cccc}
\text { Let } & \varepsilon \text { be sufficiently small such } \\
\left(a_{11}+\frac{c_{1} d_{1}}{e_{1}}\right)\left(r_{2}-0.5 \sigma_{2}^{2}-\varepsilon\right)-a_{21}\left(r_{1}-0.5 \sigma_{1}^{2}+\varepsilon\right)>0, & \text { we can }
\end{array}
$$

that

obtain from (25)

$$
-a_{21}\left[t^{-1} \ln x_{1}(t)\right]^{*} \geq\left(a_{11}+e_{1}^{-1} c_{1} d_{1}\right)\left(r_{2}-0.5 \sigma_{2}^{2}-\varepsilon\right)-a_{21}\left(r_{1}-0.5 \sigma_{1}^{2}+\varepsilon\right)>0
$$

Make use of the arbitrariness of $\varepsilon$ and Lemma 3.3, we can obtain

$$
\lim _{t \rightarrow+\infty} x_{1}(t)=0, \quad \lim _{t \rightarrow+\infty} u_{1}(t)=0, \quad \text { a.s. }
$$

At the same time, the similar proof of (II), we can obtain

$\lim _{t \rightarrow+\infty} t^{-1} \int_{0}^{t} x_{2}(s) d s=\frac{\left(r_{2}-0.5 \sigma_{2}^{2}\right) e_{2}}{a_{22} e_{2}+c_{2} d_{2}}, \lim _{t \rightarrow+\infty} t^{-1} \int_{0}^{t} u_{2}(s) d s=\frac{\left(r_{2}-0.5 \sigma_{2}^{2}\right) d_{2}}{a_{22} e_{2}+c_{2} d_{2}}$ a.s.

So we omitted it in here.

(b) According to (19), we can see

$a_{21}\left\langle x_{1}(t)\right\rangle+\left(a_{22}+e_{2}^{-1} c_{2} d_{2}\right)\left\langle x_{2}(t)\right\rangle+t^{-1} \ln x_{2}(t) \geq\left(r_{2}-0.5 \sigma_{2}^{2}-\varepsilon\right)+t^{-1} \sigma_{2} B_{2}(t)$

by virtue of $\left[t^{-1} \ln x_{2}(t)\right]^{*} \leq 0$, we can obtain

$a_{21}\left\langle x_{1}(t)\right\rangle^{*}+\left(a_{22}+e_{2}^{-1} c_{2} d_{2}\right)\left\langle x_{2}(t)\right\rangle^{*} \geq\left(r_{2}-0.5 \sigma_{2}^{2}\right)>0$

So the fact that is ${ }^{x_{1}}$ and ${ }^{x_{2}}$ not simultaneously go to extinction from (26).

\section{REFERENCES}

[1] K. Gopalsamy, P.X. Weng, Feedback regulation of logistic growth, Int. J. Math. Sci. 16 (1993) 177-192.

[2] S. Lefschetz, Stability of Nonlinear Control Systems, Academic Press, New York, 1965.

[3] K. Gopalsamy, P.X. Weng, Global attractivity in a competition system with feedback controls, Comput. Math. Appl. 45 (2003) $665-$ 676.

[4] A. Bahar, X. Mao, Stochastic delay population dynamics, Int. J. Pure Appl. Math. 11 (2004) 377-400.

[5] L.C. Hung, Stochastic delay population systems, Appl. Anal. 88 (2009) 1303-1320.

[6] M. Liu, K. Wang, Extinction and permanence in a stochastic nonautonomous population system, Appl. Math. Lett. 23 (2010) 14641467.

[7] M. Liu, K. Wang, Survival analysis of a stochastic cooperation system in a polluted environment, J. Biol. Systems 19 (2011) 183-204.

[8] M. Liu, K. Wang, Stationary distribution, ergodicity and extinction of a stochastic generalized logistic system, Appl. Math. Lett. 25 (2012) 1980-1985.

[9] M. Liu, k. Wang, Q. Wu, Survival Analysis of Stochastic Competitive Models in a Polluted Environment and Stochastic Competitive Exclusion Principle. Bull Math Biol (2011) 73: 1969-2012

[10] M. Liu, K. Wang, A note on a delay Lotka-Volterra competitive system with random perturbations, Appl. Math. Lett. 26 (2013) 589594.

[11] C.Y. Ji, D.Q. Jiang, N.Z. Shi, Analysis of a predator-prey model with modified Leslie-Gower and Holling-type II schemes with stochastic perturbation, J. Math. Anal. Appl. 359 (2009) 482-498.

[12] X. Li, X. Mao, Population dynamical behavior of non-autonomous Lotka-Volterra competitive system with random perturbation, Discrete Contin. Dyn. Syst. 24 (2009) 523-545.

[13] Gopalsamy K. Global asymptotic stability in Volterra's population systems. J Math Biol 1984; 19: 68-157.

[14] M.L. Zeeman, Extinction in competitive Lotka-Volterra systems, Proc. Amer. Math. Soc. 123 (1995) 87-96. 